\title{
REVIEW
}

\section{Cerebral perfusion in sepsis}

\author{
Christoph S Burkhart'1, Martin Siegemund² and Luzius A Steiner*3 \\ This article is one of ten reviews selected from the Yearbook of Intensive Care and Emergency Medicine 2010 (Springer Verlag) and co-published \\ as a series in Critical Care. Other articles in the series can be found online at http://ccforum/series/yearbook. Further information about the \\ Yearbook of Intensive Care and Emergency Medicine is available from http://www.springer.com/series/2855.
}

\section{Introduction}

Sepsis, the host's reaction to infection, characteristically includes multi-organ dysfunction. Brain dysfunction is often one of the first clinical symptoms in sepsis and may manifest as sepsis-associated delirium in up to $70 \%$ of patients [1,2], less often as focal deficits or seizures [3]. As severely reduced global perfusion leading to hypotension, maldistribution of regional blood flow, and tissue hypoperfusion is a key feature of severe sepsis and septic shock, the question whether there is a link between cerebral perfusion and brain dysfunction in sepsis is obvious. However, clinical and experimental data on cerebral perfusion in sepsis are often inconsistent and most reports only include small numbers of animals or patients. We summarize the current literature on the effects of the inflammatory response on cerebral perfusion and review the effects of altered cerebral perfusion on brain function in sepsis.

\section{Sepsis and the brain}

In sepsis, the brain may be affected by many systemic disturbances, such as hypotension, hypoxemia, hyperglycemia, hypoglycemia, and organ dysfunction (e.g., increased levels of ammonia in liver dysfunction or urea in acute kidney injury). Direct brain pathologies, such as ischemic brain lesions, cerebral micro- and macrohemorrhage, microthrombi, microabscesses, and multifocal necrotizing leukencephalopathy, have also been described in histopathologic examinations $[4,5]$. However, in addition to these metabolic and 'mechanical' effects on the brain, inflammation by itself causes profound alterations in cerebral homeostasis in sepsis.

*Correspondence: luzius.steiner@chuv.ch

${ }^{3}$ Department of Anesthesiology, Centre Hospitalier Universitaire Vaudois, Rue du Bugnon 46, 1011 Lausanne, Switzerland

Full list of author information is available at the end of the article

\section{Inflammation and the brain}

Sepsis at the outset causes a hyperinflammatory reaction, followed by a counteractive anti-inflammatory reaction. Pro- and anti-inflammatory cytokines are initially upregulated. Despite its anatomical sequestration from the immune system by the blood-brain barrier, the lack of a lymphatic system, and a low expression of histocompatibility complex antigens, the brain is not isolated from the inflammatory processes occurring elsewhere in the body. The circumventricular organs lack a bloodbrain barrier, and through these specific brain regions blood-borne cytokines enter the brain $[5,6]$. The circumventricular organs are composed of specialized tissue and are located in the midline ventricular system. They consist of the organum vas culosum, the pineal body, the subcommissural organ, and the subfornical organ. They also express components of the immune system (Toll-like receptors [TLR]), and receptors for cytokines such as interleukin-1 $\beta$ (IL-1 $\beta$ ), interleukin-6 (IL-6), and tumor necrosis factor- $\alpha$ (TNF- $\alpha$ ).

A further mechanism by which the brain can detect systemic inflammation is through afferent vagal fibers ending in the nucleus tractus solitarius, which senses visceral inflammation through its axonal cytokine receptors. In response to the detection of systemic inflammation, behavioral, neuroendocrine, and autonomic responses are generated including expression of immune receptors and cytokines, inducible nitric oxide synthase (iNOS), and prostaglandins leading to oxidative stress, mitochondrial dysfunction, and apoptosis $[5,7,8]$.

\section{Effects of sepsis on the blood-brain barrier and the vascular endothelium}

The blood-brain barrier, established by the tight junctions of the endothelial cells in interaction with astrocytic foot processes and pericytes, is responsible for a tightly regulated microenvironment in the brain. It prevents circulating noxious substances from entering into the 
brain and regulates brain capillary blood flow [1]. In sepsis, cerebral endothelial cells are activated by lipopolysaccharide (LPS) and pro-inflammatory cytokines, including bradykinin, IL-1 $\beta$, and TNF- $\alpha$; TNF- $\alpha$ also activates iNOS [9]. These changes in the cerebral microcirculation are associated with the upregulation of mRNA for local production of IL-1 $\beta$, TNF- $\alpha$, IL- 6 , and NO by induction of iNOS. Furthermore, leukocytes stick to the wall of blood vessels and enter the brain, mediated by adhesion molecules. The expression of one such adhesion molecule, the intercellular adhesion molecule (ICAM), is increased in septic rats [10]. These local factors can promote endothelial dysfunction and result in blood-brain barrier breakdown leading to an increased permeability of the blood-brain barrier and to perivascular edema, as has been demonstrated in several animal models of sepsis [11-13]. The former facilitates the passage of neurotoxic factors, while the latter impairs the passage of oxygen, nutrients, and metabolites. The increased diapedesis of leukocytes and the perivascular edema decrease microcirculatory blood flow in the brain capillaries. Further evidence for an alteration in the blood-brain barrier comes from work by Alexander and colleagues [14]. In an animal model, these authors demonstrated that endotoxemia-triggered inflammation in the brain led to an alteration in the blood-brain barrier, including an upregulation of aquaporin 4 and associated brain edema. This sequence of events appeared to be mediated by TNF- $\alpha$ signaling through the TNF receptor 1 [14].

In a recent magnetic resonance imaging (MRI) study in nine humans with septic shock and brain dysfunction, sepsis-induced lesions could be documented in the white matter suggesting blood-brain barrier breakdown [15]. However, in a pathologic study no evidence of cerebral edema was reported in 23 patients who died of septic shock [4].

NO is produced by the endothelium and plays an important role in the regulation of vascular tone; its increased release may be responsible for the vasodilatation and hypotension in sepsis [16]. iNOS is activated by endotoxins and cytokines leading to local and general vasodilatation $[8,17,18]$. NO is also considered a potent cerebral vasodilator [19]. Thus, NO may play an important role, not only in mediating systemic vascular resistance, hypotension, and cardiac depression, but also in cerebral vasodilatation during sepsis. However, in an ovine model of hypotensive-hyperdynamic sepsis, Booke and colleagues [20] demonstrated that inhibition of NOS did not alter cerebral blood flow $(\mathrm{CBF})$ and postulated that $\mathrm{CBF}$ is regulated by mechanisms other than NO during sepsis. Nonetheless, in situations of ischemia and reperfusion the presence of great amounts of NO can cause an increased production of reactive oxygen species
(ROS), like peroxynitrite, responsible for the destruction of membranes in cells and mitochondria.

Finally, another mechanism by which the brain is affected in sepsis is the generation of ROS by activated leukocytes. Exposed to these radicals, erythrocyte cell membranes become less deformable and may be unable to enter the brain microcirculation, thus aggravating the cerebral hypoperfusion seen in sepsis [21,22]. The brain itself with its high oxygen consumption and low antioxidant defense is susceptible to damage by ROS. Generation of ROS may alter oxidative phosphorylation and cytochrome activity in the mitochondria and impair cerebral energy production.

\section{Cerebral perfusion}

\section{Cerebral perfusion pressure}

Mean arterial pressure (MAP) is notoriously low in severe sepsis and septic shock. Accordingly, cerebral perfusion pressure (CPP) is low. Moreover, in view of the possible presence of brain edema, the influence of intracranial pressure (ICP) on CPP must be considered. Pfister et al. [23] measured ICP non-invasively in 16 patients with sepsis and reported moderate elevations of ICP > $15 \mathrm{mmHg}$ in $47 \%$ of patients; an increase $>20 \mathrm{mmHg}$ was not observed. $\mathrm{CPP}<50 \mathrm{mmHg}$ was found in $20 \%$ of their patients. Assuming that cerebrovascular pressure autoregulation is intact and the plateau of the autoregulatory curve is not shifted, their results suggest that CPP in the majority of the patients they investigated was likely to remain in the lower range of the autoregulatory plateau. However, this interpretation is partially in contrast to measurements of CBF in patients with sepsis. Bowton et al. [21] demonstrated that CBF was reduced in patients with sepsis independent from changes in blood pressure or cardiac output. These authors used the ${ }^{133} \mathrm{Xe}$ clearance technique to measure CBF in nine septic patients. Similarly, Maekawa et al. [22] found significantly lower CBF in six patients with sepsisassociated delirium than in awake controls. In an experimental model of human endotoxemia, Moller and colleagues [24] reported a reduction in CBF after an intravenous bolus of endotoxin in healthy volunteers. However, the authors assumed that $\mathrm{CO}_{2}$ reactivity was intact in their subjects and explained this CBF reduction to hypocapnia occurring because of general symptoms of malaise, although they did not measure $\mathrm{CO}_{2}$ reactivity in their subjects.

\section{Regulation of cerebral perfusion $\mathrm{CO}_{2}$-reactivity}

Using transcranial Doppler (TCD) and arterial partial pressure of $\mathrm{CO}_{2}\left(\mathrm{PaCO}_{2}\right)$ levels between 3.0 and $7.0 \mathrm{kPa}$, Matta and Stow [25] found relative $\mathrm{CO}_{2}$-reactivity to be within normal limits in ten patients with sepsis. Their 
patients were in the early stages of sepsis $(<24 \mathrm{~h}$ after admission to ICU), were all mechanically ventilated, and received infusions of midazolam and fentanyl. Absolute $\mathrm{CO}_{2}$-reactivity was lower than had been reported in subjects who were awake but consistent with values obtained during sedation and anesthesia. Similarly, Thees and colleagues [26] reported a normal response to a decrease in $\mathrm{PaCO}_{2}$ in ten patients with sepsis using TCD and cardiac output measurement by thermal dilution. Their patients were all mechanically ventilated, and sepsis had been established for $>48 \mathrm{~h}$. Bowton and colleagues [21] also reported normal specific reactivity of the cerebral vasculature to changes in $\mathrm{CO}_{2}$ in nine septic patients. However, Terborg and colleagues [27] reported impaired $\mathrm{CO}_{2}$-reactivity in septic patients, independent of changes in MAP. They used TCD and near-infrared spectroscopy (NIRS) to assess $\mathrm{CO}_{2}$-induced vasomotor reactivity by inducing hypercapnia through reductions in the ventilatory minute volume in eight mechanically ventilated septic patients. It is important to note that all their patients suffered from a neurological or neurosurgical illness, which may have affected the results. Similarly, Bowie and colleagues [28] observed significantly impaired cerebral $\mathrm{CO}_{2}$-reactivity in septic patients in a study of 12 sedated and ventilated patients who had sepsis for $>24 \mathrm{~h}$ using TCD at normocapnia, hypocapnia, and hypercapnia. The small sample sizes, differences in timing of the measurements of $\mathrm{CO}_{2}$-reactivity and in the severity of illness between groups, which is reflected by the significant differences in mortality as well as in some of the drugs used in the management of these patients, may be responsible for the conflicting findings.

\section{Cerebrovascular pressure autoregulation}

Only a few studies have addressed the effects of sepsis on cerebral autoregulation. Matta and Stow [25] reported intact pressure autoregulation in ten mechanically ventilated patients with sepsis (not in septic shock) using a phenylephrine infusion to increase MAP by $20 \mathrm{mmHg}$ and calculated an index of autoregulation by dividing the percentage change in estimated cerebral vascular resistance by the percentage change in MAP. Conversely, Smith and colleagues [29] reported loss of cerebrovascular autoregulation in 15 patients with septic shock as they were able to demonstrate a correlation between cardiac index and CBF using TCD and cardiac output measured by thermodilution. In a recent study, Pfister and colleagues [30, 31] found disturbed cerebral autoregulation in patients with sepsis-associated delirium but not in patients with 'plain' sepsis - using TCD and NIRS. This suggests that cerebral autoregulation is possibly intact in patients with sepsis but disturbed with more severe disease or complications manifesting as septic shock or sepsis-associated delirium.

\section{Perfusion and brain dysfunction Cerebral ischemia}

Cerebral ischemia is a reality in sepsis: In a post-mortem analysis of the brain of patients who died from sepsis, multiple small ischemic lesions could be identified in different areas of the brain [4]. Possible explanations are the hypotension seen in sepsis, especially when concurrent with preexisting cerebrovascular disease or autoregulatory failure. Thrombotic mechanisms due to a high hematocrit and increased viscosity of blood in sepsis may lead to watershed infarction as has been described in a septic patient with prolonged hypotension [3].

\section{Cerebral perfusion and sepsis-associated delirium}

Sepsis-associated delirium is a common organ dysfunction in sepsis and may actually occur before failure of other organs. It can be found in up to $70 \%$ of patients with the sepsis syndrome and is correlated with the severity of sepsis [32-34]. Depending on the criteria used for diagnosis, it may be detected in almost all patients with sepsis [32, 35]. Sepsis-associated delirium has been reported as an independent predictor of death [36]; however it may only reflect the severity of illness and may not be the cause of death itself. Sepsis-associated delirium presents as an alteration of the mental state and may range from lethargy or mild disorientation to obtundation and coma. The pathophysiology of sepsisassociated delirium is incompletely understood and is probably multifactorial. Mechanisms postulated to cause sepsis-associated delirium include brain activation by inflammatory mediators via the vagus nerve and the circumventricular organs, which interfere with the liberation of neurotransmitters and neurohormones. Oxidative stress and formation of ROS compromising cell function and endothelial activation resulting in disruption of the blood-brain-barrier are other mechanisms proposed to play a role in development of sepsisassociated delirium [5]. However, cerebrovascular autoregulation may also play a role in sepsis-associated delirium [25, 27, 29, 30, 36]. Pfister and colleagues [30] reported less efficient autoregulation in patients with sepsis-associated delirium compared to patients without sepsis-associated delirium. However, in the same patients, cerebral oxygenation measured by NIRS did not differ between patients with and without sepsisassociated delirium. Thus, reduced cerebral blood flow and disturbed cerebrovascular autoregulation may among others - be important precipitating factors for sepsis-associated delirium [2, 30]. Alternatively, it could also be argued that disturbed autoregulation is merely a reflection of a more severe inflammatory stimulus that is associated with a more profound dysfunction of the blood-brain barrier and hence endothelial/autoregulatory dysfunction. 


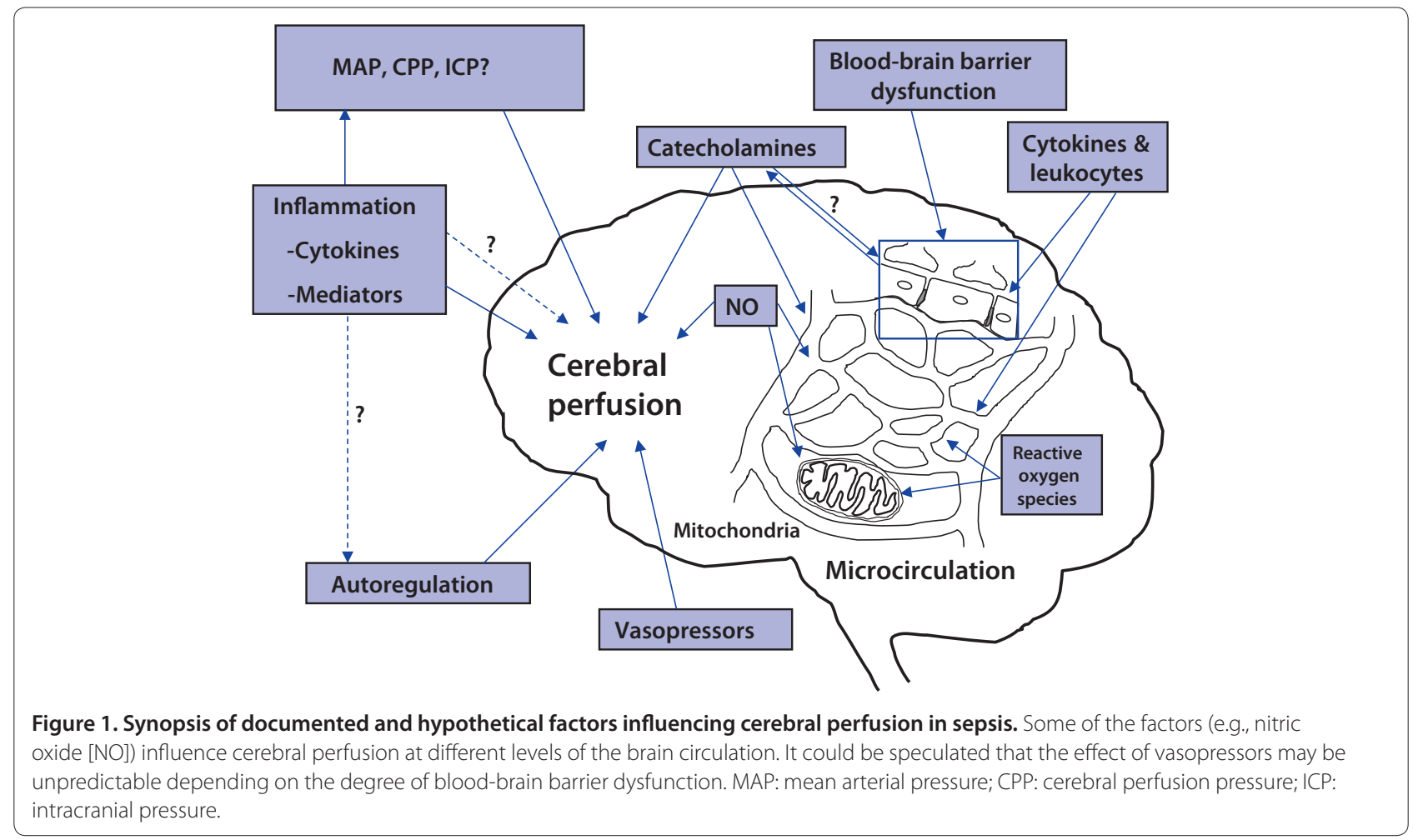

\section{Effects of catecholamines on cerebral perfusion in patients with sepsis}

Data on the cerebrovascular effects of catecholamines in sepsis are scarce. The blood-brain barrier prevents catecholamines from entering the brain as long as it is intact. Cerebral hemodynamics are not directly affected by norepinephrine and phenylephrine in anesthetized patients without cerebral pathology [37]. After head injury however, dopamine, norepinephrine and phenylephrine all seem to increase CBF with the effect of norepinephrine being more predictable than that of dopamine [38]. This is possibly due to the fact that in head injury there is also a disruption of the blood-brain barrier that allows, e.g., norepinephrine to access intracerebral $\beta$ receptors leading to an increase in cerebral metabolism and, hence, CBF [39]. Accordingly, it could be speculated that in sepsis also the cerebral effects of vasopressors may be unpredictable depending on the degree of blood-brain barrier dysfunction.

A representation of documented and hypothetical factors influencing cerebral perfusion in sepsis is shown in Figure 1.

\section{Conclusion}

The inflammatory response observed in sepsis triggers profound changes in the brain. Blood-brain barrier permeability is increased, and substantial changes in regulation of $\mathrm{CBF}$ and cerebral perfusion may occur.
Hypoperfusion due to severe hemodynamic instability will obviously lead to ischemic brain injury. Furthermore, the changes in pressure autoregulation may result in an increased vulnerability of the brain to hypoperfusion. However, this does not explain the full range of brain dysfunction found in septic patients. So far it has not been possible to establish a clear link between cerebral perfusion and sepsis-associated delirium. It is conceivable that the effects of the inflammatory response on the brain per se are the key events leading to sepsis-associated delirium, and that the observed changes in CBF regulation are rather a consequence of inflammation than a cause of sepsis-associated delirium.

\section{Abbreviations}

$\mathrm{CBF}=$ cerebral blood flow, $\mathrm{CPP}=$ cerebral perfusion pressure, $\mathrm{ICAM}=$ intercellular adhesion molecule, ICP = intracranial pressure dysfunction, ICU = intensive care unit, $\mathrm{L}$ = interleukin, $\mathrm{NNOS}=$ inducible nitric oxide synthase, $\mathrm{LPS}=$ lipopolysaccharide, $\mathrm{MAP}=$ mean arterial pressure, $\mathrm{MRI}=$ magnetic resonance imaging, $\mathrm{NIRS}=$ near-infrared spectroscopy, $\mathrm{NO}=$ nitric oxide, $\mathrm{PaCO}_{2}=$ arterial partial pressure of $\mathrm{CO}_{2^{\prime}} \mathrm{ROS}=$ reactive oxygen species, $\mathrm{TCD}=$ transcranial Doppler, $T L R=$ Toll-like receptors, $T N F=$ tumour necrosis factor.

\section{Acknowledgments}

We would like to thank Allison Dwileski, BS for her expert assistance in the preparation of this manuscript.

\section{Author details}

'Department of Anesthesia and Intensive Care Medicine, University Hospital, Spitalstrasse 21, 4031 Basel, Switzerland

2Department of Anesthesia and Intensive Care Medicine, Operative Intensive Care Unit, University Hospital, Spitalstrasse 21, 4031 Basel, Switzerland ${ }^{3}$ Department of Anesthesiology, Centre Hospitalier Universitaire Vaudois, Rue du Bugnon 46, 1011 Lausanne, Switzerland 


\section{Competing interests}

The authors declare that they have no competing interests.

Published: 9 March 2010

\section{References}

1. Pytel P, Alexander JJ: Pathogenesis of septic encephalopathy. Curr Opin Neurol 2009, 22:283-287.

2. Papadopoulos MC, Davies DC, Moss RF, Tighe D, Bennett ED: Pathophysiology of septic encephalopathy: a review. Crit Care Med 2000, 28:3019-3024.

3. Nagaratnam N, Brakoulias V, Ng K: Multiple cerebral infarcts following septic shock. J Clin Neurosci 2002, 9:473-476.

4. Sharshar T, Annane D, de la Grandmaison GL, Brouland JP, Hopkinson NS, Francoise G: The neuropathology of septic shock. Brain Pathol 2004, 14:21-33.

5. Siami S, Annane D, Sharshar T: The encephalopathy in sepsis. Crit Care Clin 2008, 24:67-82.

6. Roth J, Harre EM, Rummel C, Gerstberger R, Hubschle T: Signaling the brain in systemic inflammation: role of sensory circumventricular organs. Front Biosci 2004 „9:290-300

7. Sharshar T, Gray F, Lorin de la Grandmaison G, et al:: Apoptosis of neurons in cardio vascular autonomic centres triggered by inducible nitric oxide synthase after death from septic shock. Lancet 2003, 362:1799-1805.

8. Wong ML, Bongiorno PB, Rettori V, McCann SM, Licinio J: Interleukin (IL) 1 beta, IL-1 receptor antagonist, IL-10, and IL-13 gene expression in the central nervous system and anterior pituitary during systemic inflammation: pathophysiological implications. Proc Natl Acad Sci USA 1997, 94:227-232

9. Freyer D, Manz R, Ziegenhorn A, et al:: Cerebral endothelial cells release TNF-alpha after stimulation with cell walls of Streptococcus pneumoniae and regulate inducible nitric oxide synthase and ICAM-1 expression via autocrine loops. J Immuno/ 1999, 163:4308-4314.

10. Hofer S, Bopp C, Hoerner C, et al:: Injury of the blood brain barrier and upregulation of icam-1 in polymicrobial sepsis. J Surg Res 2008, 146:276-281.

11. Papadopoulos MC, Lamb FJ, Moss RF, Davies DC, Tighe D, Bennett ED: Faecal peritonitis causes oedema and neuronal injury in pig cerebral cortex. Clin Sci (Lond) 1999, 96:461-466.

12. Sharshar T, Hopkinson NS, Orlikowski D, Annane D: Science review: The brain in sepsis-culprit and victim. Crit Care 2005, 9:37-44.

13. Ari I, Kafa IM, Kurt MA: Perimicrovascular edema in the frontal cortex in a rat model of intraperitoneal sepsis. Exp Neurol 2006, 198:242-249.

14. Alexander JJ, Jacob A, Cunningham P, Hensley L, Quigg RJ: TNF is a key mediator of septic encephalopathy acting through its receptor, TNF receptor-1. Neurochem Int 2008, 52:447-456.

15. Sharshar T, Carlier R, Bernard F, et al:: Brain lesions in septic shock: a magnetic resonance imaging study. Intensive Care Med 2007, 33: 798-806.

16. Moncada S, Palmer RM, Higgs EA: Nitric oxide: physiology, pathophysiology, and pharmacology. Pharmacol Rev 1991 43:109-142.

17. Avontuur JA, Bruining HA, Ince C: Nitric oxide causes dysfunction of coronary autoregulation in endotoxemic rats. Cardiovasc Res 1997. 35:368-376

18. Szabo C: Alterations in nitric oxide production in various forms of circulatory shock. New Horiz 1995, 3:2-32.

19. Marshall JJ, Wei EP, Kontos HA: Independent blockade of cerebral vasodilation from acetylcholine and nitric oxide. Am J Physiol 1988, 255:H847-854.
20. Booke M, Westphal M, Hinder F, Traber LD, Traber DL: Cerebral blood flow is not altered in sheep with Pseudomonas aeruginosa sepsis treated with norepinephrine or nitric oxide synthase inhibition. Anesth Analg 2003, 96:1122-1128.

21. Bowton DL, Bertels NH, Prough DS, Stump DA: Cerebral blood flow is reduced in patients with sepsis syndrome. Crit Care Med 1989, 17:399-403.

22. Maekawa T, Fujii Y, Sadamitsu D, et al: Cerebral circulation and metabolism in patients with septic encephalopathy. Am J Emerg Med, 1991 9:139-143.

23. Pfister D, Schmidt B, Smielewski P, et al.: Intracranial pressure in patients with sepsis. Acta Neurochir Supp/ 2008, 102:71-75.

24. Moller K, Strauss GI, Qvist J, et al.: Cerebral blood flow and oxidative metabolism during human endotoxemia. J Cereb Blood Flow Metab 2002, 22:1262-1270.

25. Matta BF, Stow PJ: Sepsis-induced vasoparalysis does not involve the cerebral vasculature: indirect evidence from autoregulation and carbon dioxide reactivity studies. $\mathrm{Br} J$ Anaesth 1996, 76:790-794.

26. Thees $C$, Kaiser M, Scholz M, et al.: Cerebral haemodynamics and carbon dioxide reactivity during sepsis syndrome. Crit Care 2007, 11:R123.

27. Terborg C, Schummer W, Albrecht M, Reinhart K, Weiller C, Rother J: Dysfunction of vasomotor reactivity in severe sepsis and septic shock Intensive Care Med 2001, 27:1231-1234

28. Bowie RA, O'Connor PJ, Mahajan RP: Cerebrovascular reactivity to carbon dioxide in sepsis syndrome. Anaesthesia 2003, 58:261-265.

29. Smith SM, Padayachee S, Modaresi KB, Smithies MN, Bihari DJ: Cerebral blood flow is proportional to cardiac index in patients with septic shock. J Crit Care 1998, 13:104-109.

30. Pfister D, Siegemund M, Dell-Kuster S, et al.: Cerebral perfusion in sepsisassociated delirium. Crit Care 2008, 12:R63.

31. Steiner LA, Pfister D, Strebel SP, Radolovich D, Smielewski P, Czosnyka M Near-infrared spectroscopy can monitor dynamic cerebral autoregulation in adults. Neurocrit Care 2009, 10:122-128.

32. Ebersoldt M, Sharshar T, Annane D: Sepsis-associated delirium. Intensive Care Med 2007, 33:941-950.

33. Sprung $\mathrm{CL}$, Peduzzi PN, Shatney $\mathrm{CH}$, et al.: Impact of encephalopathy on mortality in the sepsis syndrome. The Veterans Administration Systemic Sepsis Cooperative Study Group. Crit Care Med 1990, 18:801-806.

34. Eggers V, Schilling A, KoxWJ, Spies C: [Septic encephalopathy. Diagnosis und therapy]. Anaesthesist 2003, 52:294-303.

35. Zauner C, Gendo A, Kramer L, et al:: Impaired subcortical and cortical sensory evoked potential pathways in septic patients. Crit Care Med 2002, 30:1136-1139.

36. Eidelman LA, Putterman D, Putterman C, Sprung CL: The spectrum of septic encephalopathy. Definitions, etiologies, and mortalities. JAMA 1996,275:470-473

37. Strebel SP, Kindler C, Bissonnette B, Tschaler G, Deanovic D: The impact of systemic vasoconstrictors on the cerebral circulation of anesthetized patients. Anesthesiology 1998, 89:67-72.

38. Pfister D, Strebel SP, Steiner LA: Effects of catecholamines on cerebral blood vessels in patients with traumatic brain injury. Eur J Anaesthesio/ Supp/2008, 42:98-103

39. Edvinsson L, Hardebo JE, Mackenzie ET, Owman C: Effect of exogenous noradrenaline on local cerebral blood flow after osmotic opening of the blood-brain barrier in the rat. J Physio/ 1978, 274:149-156.

doi:10.1186/cc8856

Cite this article as: Burkhart CS, et al:: Cerebral perfusion in sepsis. Critical Care 2010, 14:215. 